\title{
Protein Family Clustering for Structural Genomics
}

\section{Yongpan Yan ${ }^{1,2}$ and John Moult ${ }^{1 *}$}

${ }^{1}$ Center for Advanced Research in Biotechnology, University of Maryland Biotechnology Institute, 9600 Gudelsky Drive Rockville, MD 20850, USA

${ }^{2}$ Molecular and Cell Biology Program, University of Maryland, College Park, MD 20742, USA

\begin{abstract}
A major goal of structural genomics is the provision of a structural template for a large fraction of protein domains. The magnitude of this task depends on the number and nature of protein sequence families. With a large number of bacterial genomes now fully sequenced, it is possible to obtain improved estimates of the number and diversity of families in that kingdom. We have used an automated clustering procedure to group all sequences in a set of genomes into protein families. Benchmarking shows the clustering method is sensitive at detecting remote family members, and has a low level of false positives. This comprehensive protein family set has been used to address the following questions. (1) What is the structure coverage for currently known families? (2) How will the number of known apparent families grow as more genomes are sequenced? (3) What is a practical strategy for maximizing structure coverage in future? Our study indicates that approximately $20 \%$ of known families with three or more members currently have a representative structure. The study indicates also that the number of apparent protein families will be considerably larger than previously thought: We estimate that, by the criteria of this work, there will be about 250,000 protein families when 1000 microbial genomes have been sequenced. However, the vast majority of these families will be small, and it will be possible to obtain structural templates for $70-80 \%$ of protein domains with an achievable number of representative structures, by systematically sampling the larger families.
\end{abstract}

(C) 2005 Elsevier Ltd. All rights reserved.

Keywords: protein sequence clustering; SCOP benchmark; structural genomics; structure coverage; protein universe to one with an experimental structure. This strategy has been widely accepted. ${ }^{3-8}$ The accuracy of comparative models depends on the closeness of the evolutionary relationships they are based on, ${ }^{9}$ and is never as high as that of a high-quality X-ray structure. Nevertheless, these models are useful for many practical applications. ${ }^{10}$

The minimum number of experimental structures that will be needed in order to model all proteins using evolutionary relationships depends on the nature of protein sequence space. In particular, this number depends on how many families of evolutionarily associable proteins there are. The recent increase in fully sequenced genomes has made it possible to estimate this quantity more reliably than in the past. Here, we make use of knowledge of the full sequences for a set of 67 bacteria to obtain such an estimate.

No sequence-based method is able to detect all evolutionary relationships: experimental structure determinations reveal previously undetectable relationships in many cases. Thus, all
Abbreviations used: PSSM, position specific scoring matrix.

E-mail address of the corresponding author: jmoult@tunc.org 
sequence-based families are, in some sense, arbitrary, reflecting the effectiveness of current relationship detection algorithms rather than the number of independent evolutionary lines. From a structural genomics perspective, current methods are sufficiently powerful that they already represent very coarse-grained sampling of structure space, so that models based on one experimental structure per family are probably at the limit of useful accuracy. ${ }^{11}$ A single family will also often embrace a number of functions. ${ }^{12}$

Clustering of proteins into families has long been used as a basis for extending function annotation, and so there is a history of algorithm development. ${ }^{13-24}$ Many of the family sets have been developed for specific purposes, and there is so far no universally accepted comprehensive source. For example, Pfam A, ${ }^{24}$ one of the best established sets, uses sensitive methods to detect remote evolutionary relationships, and is curated by hand, providing a high level of reliability. As a consequence, coverage is incomplete.

We have developed an automated family classification scheme, applicable to estimation of the number of experimental structures that will be needed for structural genomics. There are three main steps: identification of evolutionary relationships; parsing of the full proteins into probable structural domains; and clustering into families. Conventional PSI-BLAST searches are used to detect sequence relationships within a set of 67 fully sequenced bacterial genomes. Lists of relationships are sub-divided on the basis of a protein domain identification method. Lists are then merged into families with a multi-linkage clustering procedure. Although a relatively standard sequence search method is used, benchmarking with SCOP structural superfamilies ${ }^{25,26}$ shows a slightly higher sensitivity than previously reported methods including profile and profile-profile methods $\uparrow .27-29$ We attribute this to the robust clustering step and reasonably effective parsing into domains.

There have been a number of studies of the number of protein families in biology. Estimates vary from 1000 to $30,000 .^{4,7,8,30-36}$ As more genome sequences are completed, it becomes possible to improve the reliability of the estimate. Our study, focusing on recently available complete genome sequences, leads to an estimate for the prokaryotes that is substantially higher than previous ones: Clustering 178,310 sequences from 67 microbial genomes already generates 31,874 families. A recent study of five fully sequenced eukaryotic genomes has also led to a much larger number than previously suggested, 45,000 protein families. ${ }^{37}$ A more relevant quantity for structural genomics is the number of detectable families there will be in future. We have developed a method of estimating growth in the number of families, and find there will be about 250,000 families when 1000 genomes

$\dagger$ http:/ / supfam.mrc-lmb.cam.ac.uk/PRC/ are sequenced. Apparent singletons (proteins with no detectable relatives) ${ }^{38}$ are the fastest growing category.

At first glance, these increased estimates are discouraging for the structural genomics goal of obtaining structures for all domains. However, because most sequences are in relatively large families, we estimate that it will still be possible to have coverage for $70 \% \sim 80 \%$ of domains within the next decade.

\section{Procedures}

\section{Protein sequences}

All identified protein sequences in 140 genomes were retrieved from Genbank $\$$. Of these, 67 were used for building the family estimate model, and the rest were reserved for testing the projections of the model. All downloaded and generated information were stored in a MySQL relational database running on a Linux server (Tables 1 and 2).

\section{Generation of homolog lists}

For each protein, a six-round PSI-BLAST search was performed against the set of all other sequences in the genome set. ${ }^{39,40}$ Low-complexity regions were omitted, using the default SEG option, ${ }^{41}$ covering $5.9 \%$ of the residues. Homologs with an $E$-value $10^{-4}$ or lower to the search sequence were collected, creating a homolog list for each protein.

\section{Domain parsing}

Each homolog list was examined for domain structures, as described below. A number of domain parsing methods have been developed. ${ }^{16,21,42}$ In the present work, domain boundaries are identified on the basis of the location of indels in the PSI-BLAST sequence alignment. Indel locations are found by counting the number of sequences with an amino acid at each position in the alignment. Figure 1 shows an example.

Domain boundaries are defined as positions in the multiple alignment where there are relatively deep minima in the number of sequences with residues. The detailed procedure is as follows.

(1) Calculate the slope of the alignment count for each position in the alignment.

(2) Find all the turning points (positions where the sign of the slope changes).

(3) Discard the trough points that make a domain too short to be viable (less than 40 residues between turning points).

(4) Discard the trough points where a trough is not significantly lower than the surroundings

$\ddagger$ http://www.ncbi.nlm.nih.gov/Genomes/index. html 
Table 1. The 67 fully sequenced microbial genomes used for protein family construction, and the number of proteins in each genome

\begin{tabular}{|c|c|}
\hline Genome & Number of proteins \\
\hline Aeropyrum pernix & 2694 \\
\hline Agrobacterium tumefaciens str. C58 (Dupont) & 5402 \\
\hline Aquifex aeolicus & 1553 \\
\hline Archaeoglobus fulgidus & 2407 \\
\hline Bacillus halodurans & 4066 \\
\hline Bacillus subtilis & 4100 \\
\hline Borrelia burgdorferi & 1637 \\
\hline Brucella melitensis & 3198 \\
\hline Buchnera sp. APS & 574 \\
\hline Campylobacter jejuni & 1629 \\
\hline Caulobacter crescentus & 3737 \\
\hline Chlamydia muridarum & 916 \\
\hline Chlamydophila pneumoniae AR39 & 1110 \\
\hline Chlamydophila pneumoniae CWL029 & 1052 \\
\hline Chlamydophila pneumoniae $\mathrm{J} 138$ & 1069 \\
\hline Clostridium acetobutylicum & 3672 \\
\hline Clostridium perfringens & 2723 \\
\hline Corynebacterium glutamicum & 3040 \\
\hline Deinococcus radiodurans & 3102 \\
\hline Escherichia coli & 4289 \\
\hline Escherichia coli O157:H7 & 5361 \\
\hline Haemophilus influenzae Rd & 1709 \\
\hline Halobacterium sp. NRC-1 & 2605 \\
\hline Helicobacter pylori 26695 & 1566 \\
\hline Helicobacter pylori $\mathrm{J} 99$ & 1490 \\
\hline Lactococcus lactis subsp. lactis & 2266 \\
\hline Listeria innocua & 3043 \\
\hline Listeria monocytogenes EGD-e & 2846 \\
\hline Mesorhizobium loti & 7275 \\
\hline Methanobacterium thermoautotrophicum & 1869 \\
\hline Methanococcus jannaschii & 1770 \\
\hline Mycobacterium leprae & 1605 \\
\hline Mycobacterium tuberculosis & 3869 \\
\hline Mycobacterium tuberculosis CDC1551 & 4187 \\
\hline Mycoplasma genitalium & 480 \\
\hline Mycoplasma pneumoniae & 688 \\
\hline Mycoplasma pulmonis & 782 \\
\hline Neisseria meningitidis & 2025 \\
\hline Neisseria meningitidis Z2491 & 2032 \\
\hline Nostoc sp. PCC 7120 & 6129 \\
\hline Pasteurella multocida & 2014 \\
\hline Pseudomonas aeruginosa & 5565 \\
\hline Pyrobaculum aerophilum & 2605 \\
\hline Pyrococcus abyssi & 1765 \\
\hline Pyrococcus horikoshii & 2064 \\
\hline Ralstonia solanacearum & 5116 \\
\hline Rhizobium sp. NGR234 & 416 \\
\hline Rickettsia conorii & 1374 \\
\hline Rickettsia prowazekii & 834 \\
\hline Salmonella enterica subsp. enterica serovar Typhi & 4749 \\
\hline Salmonella typhimurium LT2 & 4553 \\
\hline Sinorhizobium meliloti & 6205 \\
\hline Staphylococcus aureus subsp. aureus Mu50 & 2748 \\
\hline Staphylococcus aureus subsp. aureus N315 & 2624 \\
\hline Streptococcus pneumoniae & 2094 \\
\hline Streptococcus pyogenes & 1696 \\
\hline Sulfolobus solfataricus & 2977 \\
\hline Sulfolobus tokodaii & 2826 \\
\hline Synechocystis PCC6803 & 3169 \\
\hline Thermoplasma acidophilum & 1478 \\
\hline Thermoplasma volcanium & 1526 \\
\hline Thermotoga maritima & 1846 \\
\hline Treponema pallidum & 1031 \\
\hline Ureaplasma urealyticum & 611 \\
\hline Vibrio cholerae & 3828 \\
\hline Xylella fastidiosa & 2831 \\
\hline Yersinia pestis & 4039 \\
\hline
\end{tabular}

There are 12 archaea, and 55 bacterial genomes. In total, there are 178,310 protein sequences. 
Table 2. The 73 recently sequenced microbial genomes used to test the family growth projections

\begin{tabular}{|c|c|}
\hline Genome & Number of proteins \\
\hline Agrobacterium tumefaciens str. C58 (Cereon) & 5299 \\
\hline Bacillus anthracis str. Ames & 5311 \\
\hline Bacillus cereus ATCC 14579 & 5255 \\
\hline Bacteroides thetaiotaomicron VPI-5482 & 4778 \\
\hline Bifidobacterium longum NCC2705 & 1729 \\
\hline Bordetella bronchiseptica & 4994 \\
\hline Bordetella parapertussis & 4185 \\
\hline Bordetella pertussis & 3446 \\
\hline Bradyrhizobium japonicum USDA 110 & 8317 \\
\hline Brucella suis 1330 & 3264 \\
\hline Buchnera aphidicola str. Bp (Baizongiapistaciae) & 504 \\
\hline Buchnera aphidicola str. Sg (Schizaphisgraminum) & 546 \\
\hline Candidatus blochmannia floridanus & 583 \\
\hline Chlamydia trachomatis & 893 \\
\hline Chlamydophila caviae GPIC & 1005 \\
\hline Chlamydophila pneumoniae TW-183 & 1113 \\
\hline Chlorobium tepidum TLS & 2252 \\
\hline Chromobacterium violaceum ATCC 12472 & 4407 \\
\hline Clostridium tetani E88 & 2373 \\
\hline Corynebacterium efficiens YS-314 & 2950 \\
\hline Coxiella burnetii RSA 493 & 2009 \\
\hline Enterococcus faecalis V583 & 3113 \\
\hline Escherichia coli CFT073 & 5379 \\
\hline Escherichia coli O157:H7 EDL933 & 5349 \\
\hline Fusobacterium nucleatum subsp. nucleatum ATCC25586 & 2067 \\
\hline Haemophilus ducreyi $35000 \mathrm{HP}$ & 1717 \\
\hline Helicobacter hepaticus ATCC 51449 & 1875 \\
\hline Lactobacillus plantarum WCFS1 & 3009 \\
\hline Leptospira interrogans serovar lai str. 56601 & 4727 \\
\hline Methanopyrus kandleri AV19 & 1687 \\
\hline Methanosarcina acetivorans C2A & 4540 \\
\hline Methanosarcina mazei Goe1 & 3371 \\
\hline Mycobacterium bovis subsp. bovis AF2122/97 & 3920 \\
\hline Mycoplasma gallisepticum $\mathrm{R}$ & 726 \\
\hline Mycoplasma penetrans & 1037 \\
\hline Nitrosomonas europaea ATCC 19718 & 2461 \\
\hline Oceanobacillus iheyensis HTE831 & 3500 \\
\hline Pirellula sp. & 7325 \\
\hline Porphyromonas gingivalis W83 & 1909 \\
\hline Prochlorococcus marinus str. MIT 9313 & 2265 \\
\hline Prochlorococcus marinus subsp. marinus str.CCMP137 & 1882 \\
\hline Prochlorococcus marinus subsp. pastoris str.CCMP13 & 1712 \\
\hline Pseudomonas putida KT2440 & 5350 \\
\hline Pseudomonas syringae pv. tomato str. DC 3000 & 5471 \\
\hline Pyrococcus furiosus DSM 3638 & 2065 \\
\hline Salmonella enterica subsp. enterica serovar Typhi Ty2 & 4323 \\
\hline Shewanella oneidensis MR-1 & 4472 \\
\hline Shigella flexneri 2a str. 2457T & 4068 \\
\hline Shigella flexneri 2a str. 301 & 4180 \\
\hline Staphylococcus aureus subsp. aureus MW2 & 2632 \\
\hline Staphylococcus epidermidis ATCC 12228 & 2419 \\
\hline Streptococcus agalactiae $2603 \mathrm{~V} / \mathrm{R}$ & 2124 \\
\hline Streptococcus agalactiae NEM316 & 2094 \\
\hline Streptococcus mutans UA159 & 1960 \\
\hline Streptococcus pneumoniae R6 & 2043 \\
\hline Streptococcus pyogenes MGAS315 & 1865 \\
\hline Streptococcus pyogenes MGAS8232 & 1845 \\
\hline Streptococcus pyogenes SSI-1 & 1861 \\
\hline Streptomyces avermitilis MA-4680 & 7575 \\
\hline Streptomyces coelicolor A3(2) & 8154 \\
\hline Synechococcus sp. WH 8102 & 2517 \\
\hline Thermoanaerobacter tengcongensis & 2588 \\
\hline Thermosynechococcus elongatus BP-1 & 2475 \\
\hline Tropheryma whipplei str. Twist & 808 \\
\hline Tropheryma whipplei TW08/27 & 783 \\
\hline Vibrio parahaemolyticus RIMD 2210633 & 4832 \\
\hline Vibrio vulnificus ČMCP6 & 4537 \\
\hline Wigglesworthia glossinidia endosymbiont of Glossina & 611 \\
\hline Wolinella succinogenes & 503 \\
\hline Xanthomonas axonopodis pv. citri str. 306 & 4312 \\
\hline Xanthomonas campestris pv. campestris str. ATCC 339 & 4181 \\
\hline Xylella fastidiosa Temecula1 & 2036 \\
\hline Yersinia pestis KIM & 4090 \\
\hline
\end{tabular}

In all, the 140 genomes code for 405,709 proteins. 


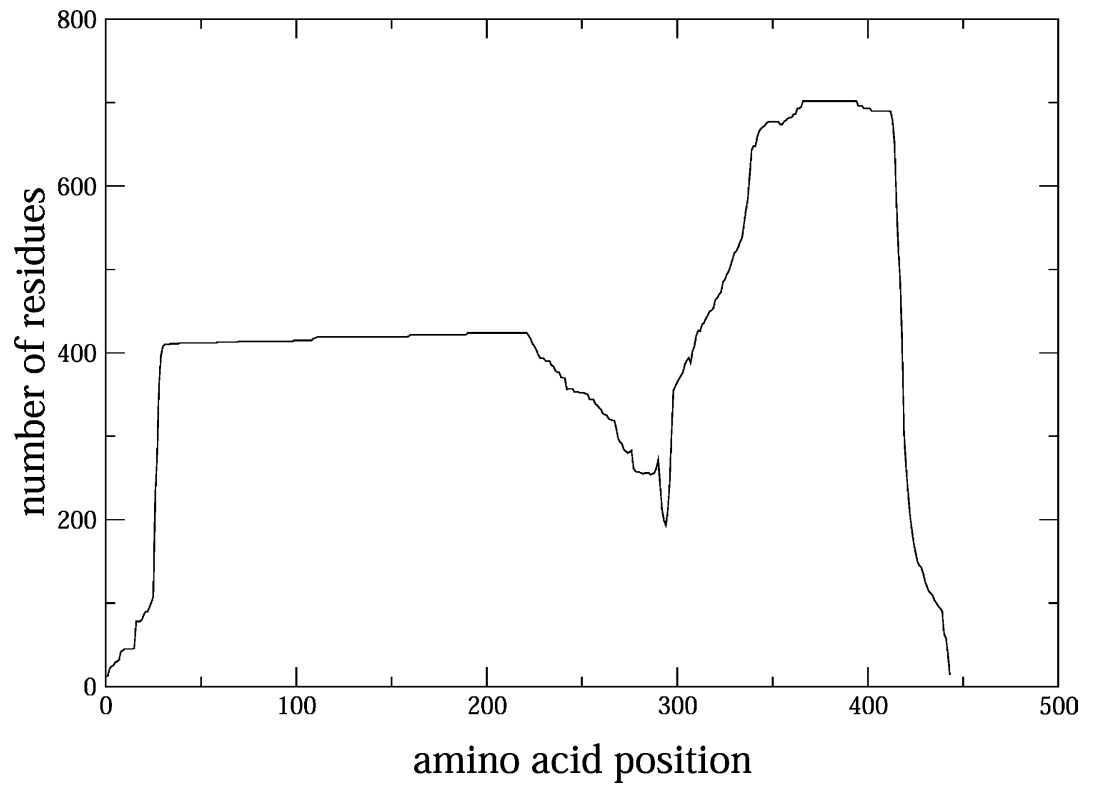

Figure 1. An example of domain parsing, for the multiple sequence alignment of E. coli ARGA (SwissProt ID P08205, amino acid acetyltransferase). The domain-splitting algorithm produces two domains, residues 20-294 and 295-443. Pfam and InterPro also split this protein into two domains. Domain 1 (26-269) belongs to Pfam PF00696, an amino acid kinase family, and domain 2 (338-414) belongs to PF00583, an acetyltransferase family. (trough height more than $60 \%$ of the peaks on either side).

(5) Divide the proteins in the homolog list into domains by cutting at each remaining trough point, to create homolog domain lists.

As described later, comparison of the results of this procedure with a set of PfamA domains in 50,000 randomly chosen Pfam sequences shows it is very conservative: $96 \%$ of single domain PfamA proteins are predicted as such, but only $24 \%$ of PfamA two domains proteins are predicted correctly. Other domain parsers adopt a different balance of false positives and false negatives. ${ }^{43}$ While this and other parsers are far from satisfactory, domain parsing does improve the quality of the families.

\section{Merging of domain lists}

Domain lists are highly redundant, in that many domains appear in multiple lists. A key step is merging of the lists to form non-redundant, domain-based protein families. Merging also increases the range of evolutionary relationships that are clustered: A PSI-BLAST search starting from protein A may find a relative $B$, but not relative $C$. On the other hand, PSI-BLAST started from protein $B$ may have found relative $C$, but not relative $A$. Merging of the $A$ and $B$ hit lists places $A$, $\mathrm{B}$ and $\mathrm{C}$ in one family.

The simplest clustering procedure is to iteratively merge all pairs of lists that contain at least one common domain, and then eliminate redundancies from the merged sets. Notoriously, this single linkage procedure leads to over-clustering, even when the false positive rate for inclusion of a domain in a single list is small. A number of strategies have been suggested for overcoming this problem. ${ }^{22,44}$ We have developed a variable linkage procedure. Short domain lists are merged on the basis of a single common entry. The longer the lists, the more common entries are required.

Merging proceeds by selecting a first list, comparing it to all others, combining where the merge rules are satisfied, then picking a next so far unconsidered list, and so on, until all lists have been considered. The process is repeated a maximum of three times.

\section{Further domain boundary checking}

Incomplete domain parsing can occasionally lead to the merging of proteins that have no significant alignment. This is illustrated in Figure 2. An unparsed, two domain protein (b) in list I has a region of alignment with protein (c) in a second list, II. Sequence (c) shares no significant relationship with the primary domain in list I, but will be merged into that list. To reduce this effect, each candidate sequence in list II is checked for alignment overlap with the first sequence in list I.

I

II

(a)

(b)
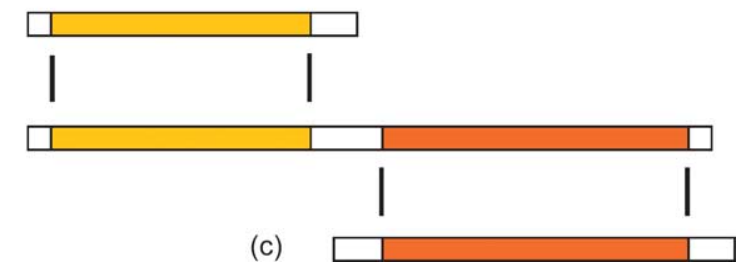

Figure 2. Domain merging check. 
List II entries with an overlap of less than 40 residues are not merged.

\section{Selection of parameter values}

Results are dependent on a number of parameters. The parameters were optimized by building a set of families for proteins with domains in PfamA, varying parameter values to maximize the agreement between the generated and Pfam families, as others have done. ${ }^{45}$ A set of 50,000 full-length SwissProt protein sequences including all the domains present in a 721 family subset of PfamA version 9.0 was used. Use of full-length protein sequences allows the domain parsing procedures to be tested.

Each generated family was compared with all the PfamA families, and the most similar one (most common sequences) was considered the best match. Two measures are used to assess the quality of the built families:

$$
\begin{aligned}
& \text { False negative fraction } F_{\mathrm{N}}=(P-O) / P \\
& \text { False positive fraction } F_{\mathrm{P}}=(M-O) / M
\end{aligned}
$$

where $P$ is the Pfam family size, $M$ is the generated family size, and $\mathrm{O}$ is the common sequences between the two. $F_{\mathrm{N}}$ is the false negative rate for a generated family; the fraction of correct domains omitted. $F_{\mathrm{P}}$ is the false positive rate for a family; the fraction of incorrect domains included.

These ratios were determined for a range of PSI-BLAST conditions, with and without domain checks, and with different linkage rules, in order to optimize the procedure. Details are given in Results.

The final choice of parameters was up to six rounds of PSI-BLAST with an E-score threshold of $10^{-4}$, and a maximum of three rounds of merging. Lists are merged into a family using the following merging rules:

(1) For lists with four or fewer members: at least one common entry required for merging.

(2) For lists with five to ten members: at least two common entries.

(3) For lists with more than ten members: at least $40 \%$ common entries.

\section{Evaluation of domain family construction}

Effectiveness of the family building procedure was assessed in terms of its ability to pair all members of SCOP superfamilies, and not to pair domains in different folds. (Note that effectiveness could not be evaluated against Pfam, since the method was tuned on that basis. SCOP $\dagger$ is a hierarchical organization of proteins based on evolutionary and structural relationships. ${ }^{25,26}$ Since structural similarity provides a much more

\footnotetext{
$\dagger$ http://scop.mrc-lmb.cam.ac.uk/scop/
}

sensitive test of evolutionary relationships between proteins than does sequence, SCOP has been widely used as a benchmark for evaluating sequence alignment, clustering, and evolutionary relationship detection methods. ${ }^{44,46}$ We have used SCOP40 (no sequence relationships higher than $40 \%$ identity) version 1.63, which contains 5226 domains, 1224 superfamilies, and 760 folds.

The 5226 domains were clustered into families, as described above: PSI-BLAST was run for each domain against the NR sequence database, augmented with the SCOP domain sequences. No domain parsing was performed, as SCOP is already domain-based. PSI-BLAST-generated homolog lists were merged using the linkage rules, to form a set of generated families.

The set of generated families was compared with the SCOP superfamilies in terms of all the possible pairwise relationships between domains. Any pair of domains found both in a generated family and a SCOP superfamily is considered a true positive. A pair of domains presented in a generated family set, but not assigned the same SCOP fold is considered a false positive, as it is unlikely to represent a homology relationship. SCOP40 version 1.63 was used, with 50,374 pairs of domains within the same superfamily, and more than 600,000 pairs of domains with each member in a different fold. True positives detected as a function of the false positives incurred were plotted in a ROC curve. A $1 \%$ false positive to true positive ratio was chosen as an overall measure of quality, as used by others. ${ }^{4-50}$

For comparison, several other alignment and family clustering methods were also tested using the same set of SCOP domains. These are BLAST, PSIBLAST, SAM-T99 (HMM) $\$, 51$ and PRC (a profile to profile method)§. Software was downloaded from the authors' web sites.

Programs and parameters used for SAM-99 were:

(1) target99-seed [sequence fasta file]-out [output file] $-\mathrm{db}[\mathrm{nr}+\mathrm{scop} 40]-$ iter 4

(2) fw0.7 [sequence.a $2 \mathrm{~m}$ file] [sequence.mod file]

(3) hmmscore [sequence name]-i [sequence.mod file] - sw 2-db [scop40]

Programs and parameters used for PRC were: Prc-Emax 10 [sequence.mod file] [mod library] [sequence name]

\section{Transmembrane protein determination}

Proteins with one or more transmembrane helical segments were identified using TMHMM $\| .^{52}$

$\ddagger$ http://www.soe.ucsc.edu/research/compbio/ sam2src/

$\S$ http:/ / supfam.mrc-lmb.cam.ac.uk/PRC/

|| http://www.cbs.dtu.dk/services/TMHMM/ 


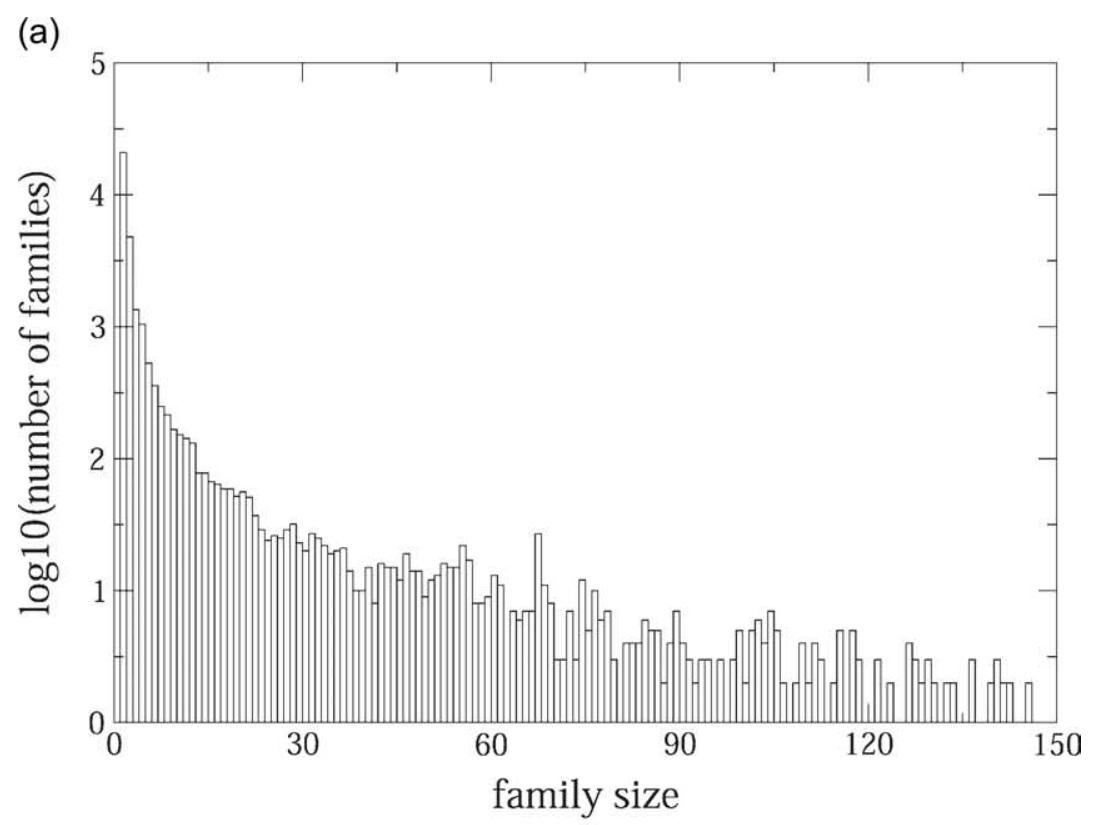

Figure 3. (a) Distribution of domain family size. Note the log scale. There is an approximately power law relationship between the number of families and family size: 20,992 of the 31,874 families have only a single member, while only 263 families are larger than 100. (b) Number of singletons (family size one), doubletons (family size two), tripletons and larger (top bar), and the percentage of sequence space covered by each of the three categories. Although there are 20,992 singletons and 4810 doubletons, these two categories represent only about $12 \%$ of sequence space. The 6072 larger families comprise the rest.

(b)

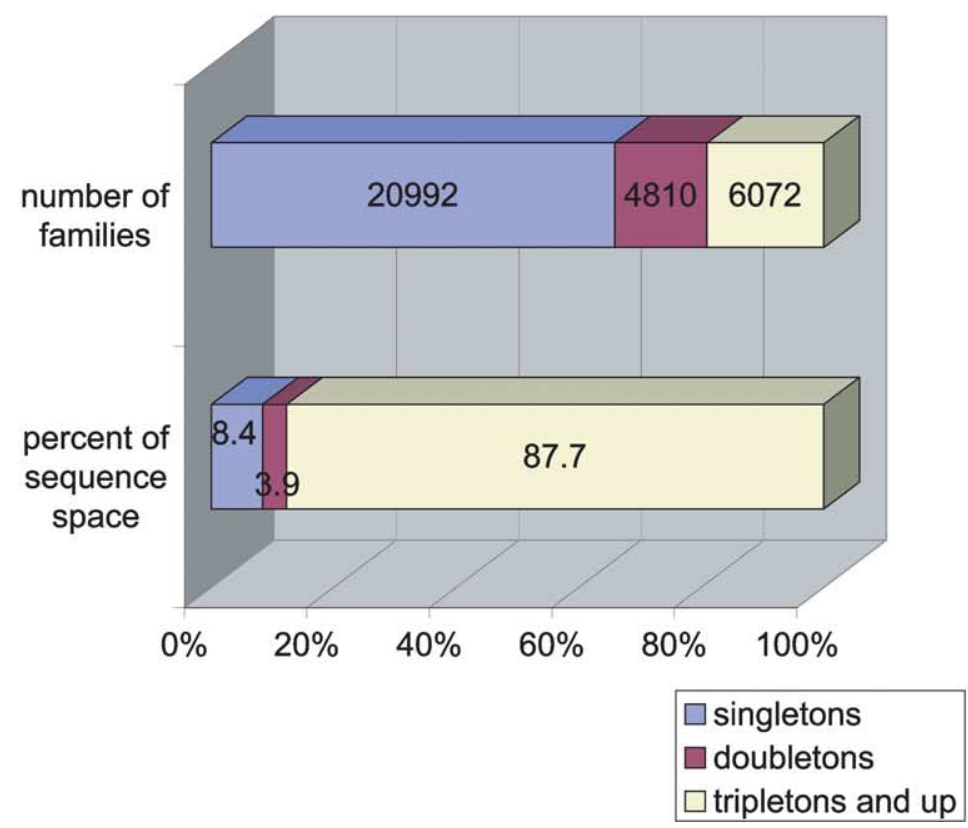

\section{Structure coverage determination}

Domain families with known structures were identified as follows. A sequence profile (position specific scoring matrix, PSSM) was obtained from the multiple sequence alignment of a protein family, using blastpgp. ${ }^{39}$ Each protein sequence in the Protein Data Bank (June 15, 2003 release) was run against the set of family sequence profiles, using RPS-BLAST ${ }^{53}$ Any profile to sequence comparison with an $E$-value of $10^{-2}$ or lower was considered to represent a family that could be modeled on the basis of the corresponding structural template. Such families were considered to be structurally covered.

\section{Results}

\section{Protein family clustering}

\section{Domain-based protein family set}

Following the clustering procedure described in Procedures, 178,310 sequences from 67 sequenced prokaryotic genomes were parsed to 249,574 domain sequences and then clustered into 31,874 sequence families. Figure 3(a) shows the distribution of family sizes. Small families predominate. There are 20,992 singletons (families with only one member), about two-thirds of the total, and 4810 
doubletons (family size two). At the other end of the spectrum, there are only 263 families larger than 100.

From the point of view of structural genomics, this result is discouraging: even this small number of genomes would require over 30,000 experimental structure determinations in order to provide templates for complete modeling. However, consideration of the large fraction of proteins in the larger families leads to a different view. Figure 3(b) compares the number of families of size one, two and larger with the total number of domains those categories contain. Although about two-thirds of the families are singletons, they represent only $8 \%$ of the domains. Families of size three and larger contain $88 \%$ of the domains, and there are only just over 6000 of those. Thus, $88 \%$ structural coverage of these 67 genomes would be provided by about 6000 experimental structure determinations.

\section{Optimization of protein family construction}

As discussed in Procedures, parameters for protein family construction were optimized by comparison of generated families with those in PfamA. ${ }^{24}$

Families were built for 50,000 full-length protein sequences covering 721 PfamA (release 9) families. The full sequences were clustered into new families, and each such family was best matched to a PfamA family. Each generated family was compared with the corresponding PfamA one using the false positive $\left(F_{\mathrm{P}}\right)$ and false negative $\left(F_{\mathrm{N}}\right)$ fractions. The smaller these values, the better the family building procedure.

Table 3 shows the level of agreement between the generated and PfamA families as a function of the $E$-value threshold for accepting PSI-BLAST relationships. Families are obviously over-clustered with a cutoff of $10^{-2}$, judging by the high level of false positives $\left(F_{\mathrm{P}}\right)$. A threshold of $10^{-4}$ produces many fewer false positives than $10^{-2}$ and a lower number of false negatives than $10^{-6}$, so was chosen as the final value. (Final values of the other parameters were used for these tests.)

Table 4 shows the agreement between the generated and PfamA families as a function of the linking procedure, domain parsing and checking, and the number of merging rounds. A maximum of six rounds of PSI-BLAST with an E-score threshold of $10^{-4}$ was used. Three rounds of single linkage clustering with no domain processing dramatically over-clusters compared with PfamA, compressing the sequences into 285 families, as opposed to the ideal 721 , with a false positive rate of $79 \%$. Domain parsing increases the number of families to 427 , at the expense of a minor increase in false negatives, from $6.9 \%$ to $8.0 \%$. Domain checking produces a further minor improvement.

Introduction of the family size-dependent linkage scheme further improves agreement with PfamA. Three rounds of merging generate 785 families with a false positive rate of $17.9 \%$. Merging for five rounds increases the false positive rate slightly to $19.7 \%$.

On the basis of these tests, the final protocol adopted was six rounds of all against all PSI-BLAST using a $10^{-4}$ threshold, followed by three rounds of hierarchical linkage. These conditions produce 785 families, of which 278 are identical with the corresponding PfamA families, with an average false positive rate of $17.9 \%$ and a false negative rate of $7.4 \%$. PfamA families are assembled using sensitive sequence methods and are curated by hand to reduce false negatives, so that a good clustering method should have a low false negative rate, as seen here. The higher false positive rate may partly reflect the fact that Pfam does not cluster some real relationships.

Table 3. Agreement between generated and PfamA families, as a function of the PSI-BLAST E score threshold

\begin{tabular}{|c|c|c|c|c|}
\hline $\begin{array}{l}\text { PSI-BLAST E-score } \\
\text { threshold }\end{array}$ & Number of families & $F_{\mathrm{N}}$ & $F_{\mathrm{P}}$ & $\begin{array}{c}\text { Number of identical } \\
\text { families }\end{array}$ \\
\hline $10^{2}$ & 569 & 0.087 & 0.325 & 204 \\
\hline $10^{4}$ & 744 & 0.079 & 0.180 & 276 \\
\hline $10^{6}$ & 788 & 0.087 & 0.176 & 273 \\
\hline
\end{tabular}

Table 4. Agreement between generated and PfamA families as a function of linkage protocol, domain parsing and checking, and the number of rounds of merging

\begin{tabular}{lcccc}
\hline Clustering method & $\begin{array}{c}\text { Number of generated } \\
\text { families }\end{array}$ & $F_{\mathrm{N}}$ & $F_{\mathrm{P}}$ & $\begin{array}{c}\text { Number of identical } \\
\text { families }\end{array}$ \\
\hline Single linkage w/o domain splitting or & 285 & 0.069 & 0.792 & 154 \\
domain check & & & 0.080 & 0.415 \\
Single linkage w/o domain check & 427 & 0.079 & 0.377 \\
Single linkage w/o domain check & 480 & 0.074 & 0.179 & 244 \\
Hierarchical merging, three rounds & 785 & 0.079 & 0.197 & 255 \\
Hierarchical merging, five rounds & 744 & 278 \\
\hline
\end{tabular}

Domain parsing, domain checking, and hierarchical linkage all improve the quality of the generated families. On the basis of these results, a protocol of three rounds of hierarchical merging, with domain parsing and checking, was adopted. 


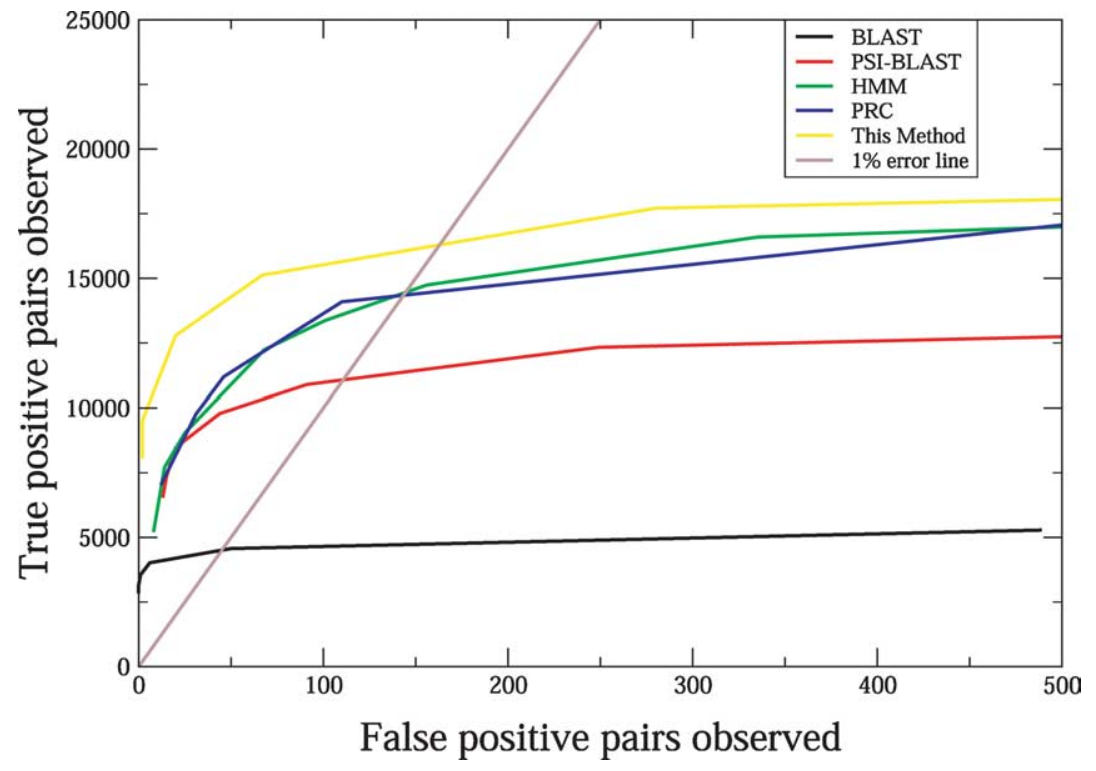

Figure 4. Benchmarking of the family building procedure, together with BLAST, PSI-BLAST, SAM-T99 and PRC, using SCOP40. True positive pairs are the fraction of pairwise relationships within superfamilies that are detected, out of 50,374 possible. False positive pairs are the fraction of apparent pairwise relationships between folds. The more true positives detected at a given false positive rate, the better the method. At a $1 \%$ ratio of false positives versus true positives, PSI-BLAST has approximately double the sensitivity of BLAST, the simple pairwise method. The hidden Markov model, SAM-T99 and the profile-profile method (PRC) improve the sensitivity to $28 \%$. The new method achieves a modest but useful improvement to $32 \%$. Improved sensitivity is attributed to the hierarchical linkage procedure.

\section{Evaluation of the protein families}

The final family building procedure was benchmarked against SCOP40 (a subset of SCOP containing no sequence identities greater than $40 \%$ ) version 1.63. The SCOP set includes 5226 domain sequences grouped into 1226 superfamilies and 760 folds. As explained in Procedures, all pairwise detected relationships between proteins in the same superfamily were considered true positives, and all apparent relationships between proteins in different folds were considered false positives. Several other methods for detecting evolutionary relationships, BLAST, PSI-BLAST, SAM-T99 (a hidden Markov model method) ${ }^{51}$ and PRC (a profile to profile method) were also evaluated.

The results are shown in Figure 4.

Overall, the new family building procedure delivers a higher fraction of true positives at a low false positive rate. At the commonly adopted threshold of $1 \%$ false positives/true positives, ${ }^{47-50}$ BLAST detects only $9 \%$ of true positives. PSIBLAST doubles the level of detection to $18 \%$. SAM-T99 and PRC both detect about $28 \%$ of true positives. Our method finds $32 \%$, a modest but useful improvement. Note that at a higher false positive rate (above 5\%, not shown in the Figure), the profile-profile method performs the best. The results for BLAST, PSI-BLAST and SAM-T99 are very similar to those obtained by Park et al. ${ }^{54}$ Their study showed that, using the PDBD40-J dataset (similar but smaller than SCOP40), BLAST is able to detect $14 \%$ of homologous relationships and the two profile methods, PSI-BLAST and SAM-T98, can detect $27 \%$ and $29 \%$, respectively.

\section{Structural genomics analysis}

\section{Structure coverage of current protein families}

A long-term aim of structural genomics is to obtain an experimentally determined structure for at least one protein in every family. We now ask to what extent that is already the case for the set of 67 bacterial protein families. We consider only families with three or more members, and exclude

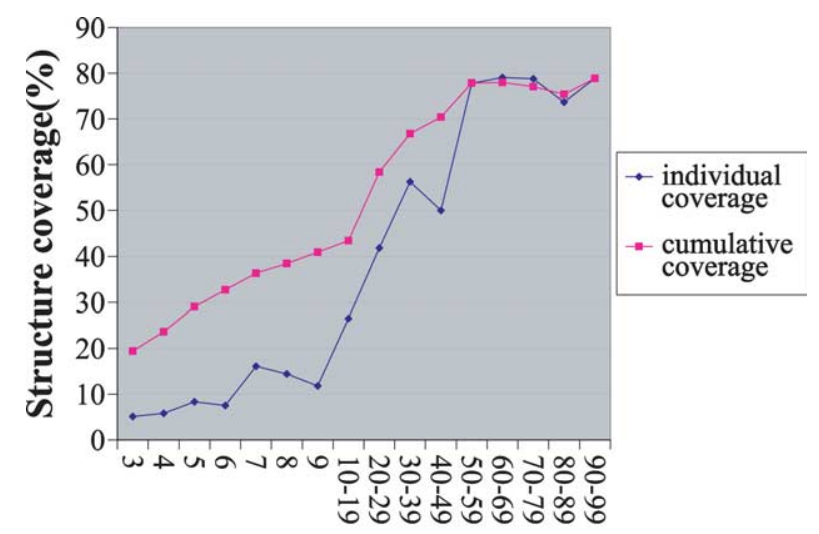

Family size

Figure 5. Fraction of the non-membrane protein families with three or more members for which there is at least one experimentally determined structure, as a function of family size (blue line). The purple line shows the coverage of all families that size and larger. Coverage is much larger for the larger families, approaching $80 \%$ for the biggest. The overall average coverage is $20 \%$. 
membrane protein families, since this class of structure is not yet amenable to high-throughput experimental techniques. There are 4907 nonmembrane protein families with three or more members. Figure 5 shows the fraction of families with one or more known structures (the structure coverage). About $80 \%$ of families larger than 60 have a structural representative. Coverage drops with decreasing family size, to around 5\% for families with only three members. Overall, 20\% of all families size three or larger have one or more representative structures. A further 3926 structures would be required to complete the coverage.

\section{Estimation of the number of families in a large number of genomes}

The previous section provides an estimate of the

(a)

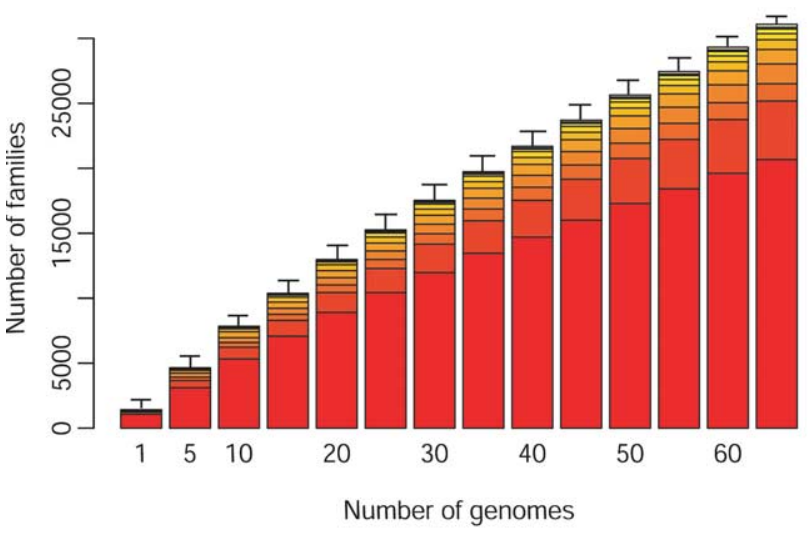

(c)

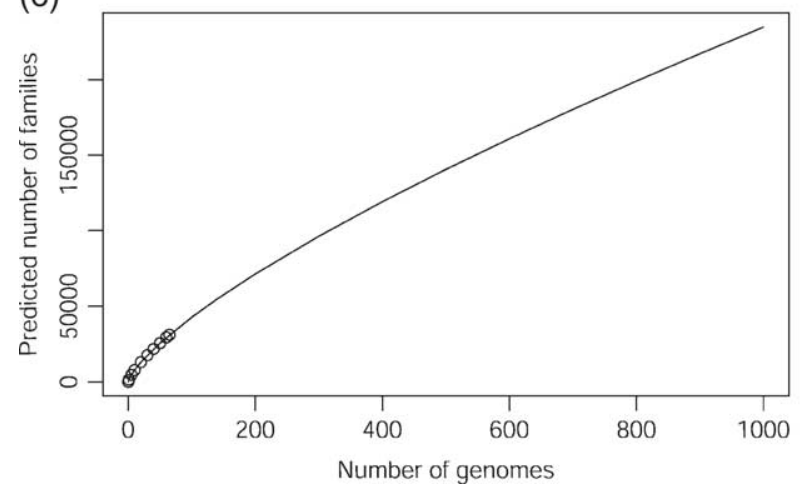

number of structures needed to complete coverage of a set of already fully sequenced genomes. Of more interest in structural genomics is the number of structures that will be needed as a function of the number of sequenced genomes and, in particular, the limit of that quantity, i.e. the number of structures that will be needed to provide coverage of all protein families.

We have examined the increase in the number of detectable protein families as the number of fully sequenced genomes increases, using the following procedure. One of the 67 prokaryotic genomes is chosen at random, and the number of families it contains noted. A second genome is selected randomly, and the additional families present in that are added. This process is continued until all 67 have been selected. The whole procedure was repeated 100 times, and the average number of

(b)

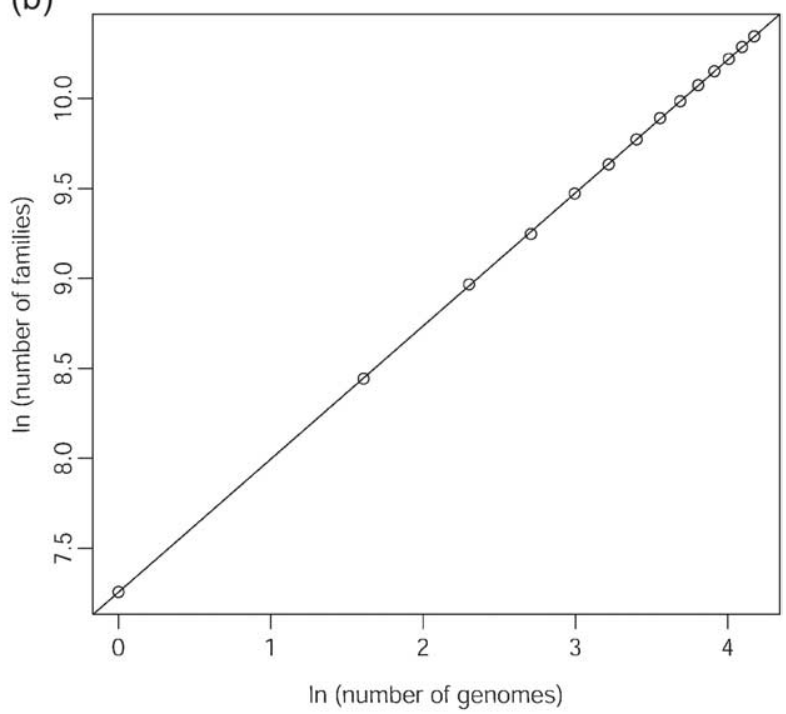

(d)

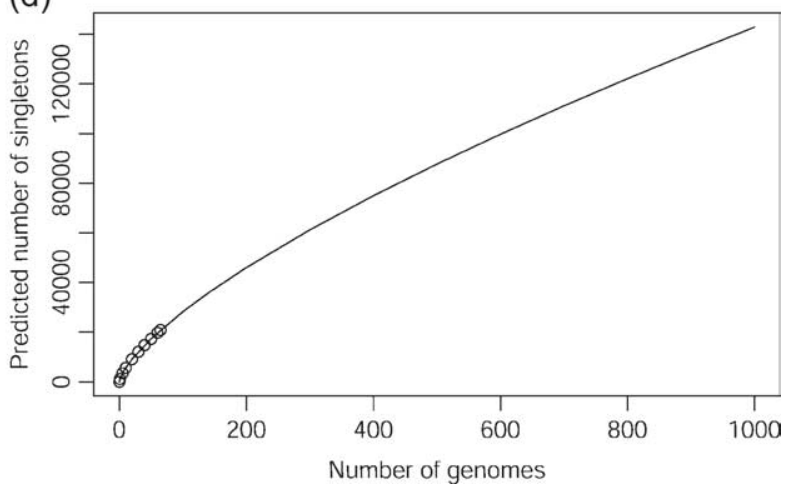

Figure 6. (a) Number of families as a function of the number of genomes. Full columns show the total families in the corresponding number of genomes, and subdivisions show the number of families in the following size ranges: $1,2,3,4$ or 5, 6-10, 11-20, 21-40, 41-70, 71-100, 101-1000. Smaller families are in the lower subdivisions. The total number of families is still increasing rapidly up to 67 genomes, and is far from saturation, though there is some decrease in the rate of growth. The singleton group is the fastest grower. The $\mathrm{T}$ bars show the standard deviations in the average number of families, over the 100 simulations. (b) Log-log view of the relationship between the number of families and number of genomes considered. A linear model gives an excellent fit to the data. (c) Predicted number of families as a function of the number of fully sequenced prokaryotic genomes, based on a log-linear fit to (b). The model predicts there will be about 250,000 families when 1000 genome sequences are available. (d) Predicted number of apparent singletons as a function of the number of fully sequenced prokaryotic genomes. The model predicts that there will be about 140,000 when the sequences of 1000 genomes are available. 
families for each number of genomes calculated. The result is shown in Figure 6(a). Total bar heights represent all families in the corresponding number of genomes. Subdivisions show the number of families in different size ranges, with smallest families lowest. The number of protein families is still growing rapidly up to inclusion of 67 genomes, and is far from saturation, though the rate of increase is slowing. Clearly there will eventually be many more than 30,000 detectable families. A log-log representation of these data (Figure $6(\mathrm{~b})$ ) is close to linear, providing a basis for extrapolation to a larger number of genomes. Figure 6(c) shows the projected number of families up to a total of 1000 genomes, using that relationship. This model predicts a total of about 250,000 families at that point, a much higher estimate than any previous ones. There is of course a limit to how far this extrapolation can be made. At some point, as most newly sequenced genomes become phylogenetically close to known ones, the number of new families must begin to decrease more rapidly. Available data for up to 140 genomes show the model holding up remarkably well (see below).

The log of the number of apparent singletons also grows approximately linearly with the log of the number of genomes, with a reliability coefficient of 0.9993 , and a slope of 0.704 . The projected number of singletons out of 1000 genomes is shown in Figure 6(d). For 1000 genomes, the estimate is 140,000 singletons.

The rapid growth of singletons in Figure 6(a) and the prediction made in Figure 6(d) clearly suggest their growth is also far from complete. This observation is contradictory to our earlier view that aggregation of homologs between genomes will lead to rapid disappearance of singletons. ${ }^{8}$

It should be borne in mind that, because of the limited sensitivity of sequence methods for detecting relationships, the large number of families does not imply a similar magnitude of independent evolutionary lines.

To test the extrapolation model, we have extended the study to include 140 prokaryotic genomes (the 67 used for the extrapolation plus 73 new ones, see Procedures) and built families for this set using the same procedure. The 405,709 sequences in these genomes produce 54,234 families, of which 36,457 are apparent singletons. The extrapolation models predict 54,910 families and 35,807 singletons, within $1 \%$ and $2 \%$, respectively, of the actual values.

\section{Structural coverage for the 67 genome set}

The previous analysis shows that it will not be possible to obtain complete structural coverage of protein family space in the near future. However, as noted earlier, a relatively small fraction of the families contain a large fraction of all the sequences. For the 67 genome analysis, $19 \%$ of the families are size three and larger, but contain $88 \%$ of the proteins. This suggests a strategy of obtaining representative structures for the largest families first. Figure 7 shows an exploration of this idea for the 67 genome set. We assume that a representative structure is first obtained for the largest family, then the next largest, and so on. The blue curve shows the result for all non-membrane protein families with three or more members. The purple curve shows the number of structures needed, taking into account the already available structures. Because of existing high coverage, very few additional ones will be needed for large families. Altogether, about 4000 structures are required to obtain complete coverage of all families with three or more members, covering $88 \%$ of the domains in these genomes. (As discussed earlier, about $20 \%$ of these

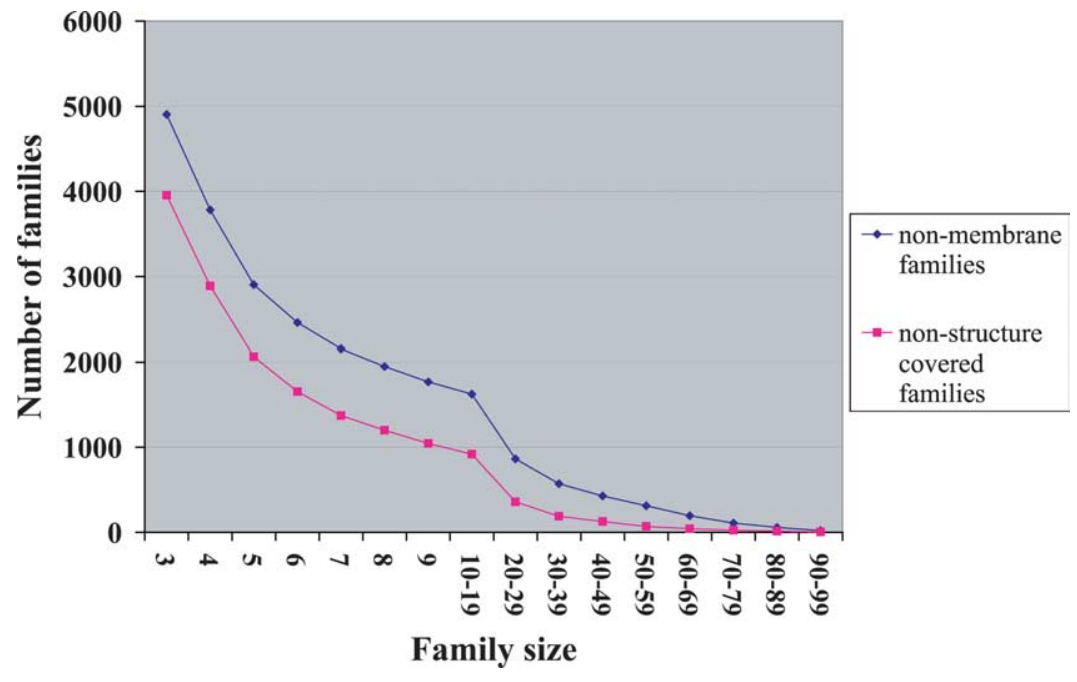

Figure 7. Cumulative number of experimental structures needed to obtain complete coverage of families size three and larger, starting with large families (right side of the plot). The blue curve is for all non-membrane protein families, and the purple curve is for the families with no current structural coverage. Very few additional structures are needed to complete coverage of large families: 1000 optimally selected ones would complete coverage of all non-membrane families larger than ten, including $80 \%$ of all the domains. About 4000 would be needed to provide one structure per family size three or larger, and would cover $88 \%$ of all the domains. (These numbers are for the set of 67 genomes analyzed in this work.) 

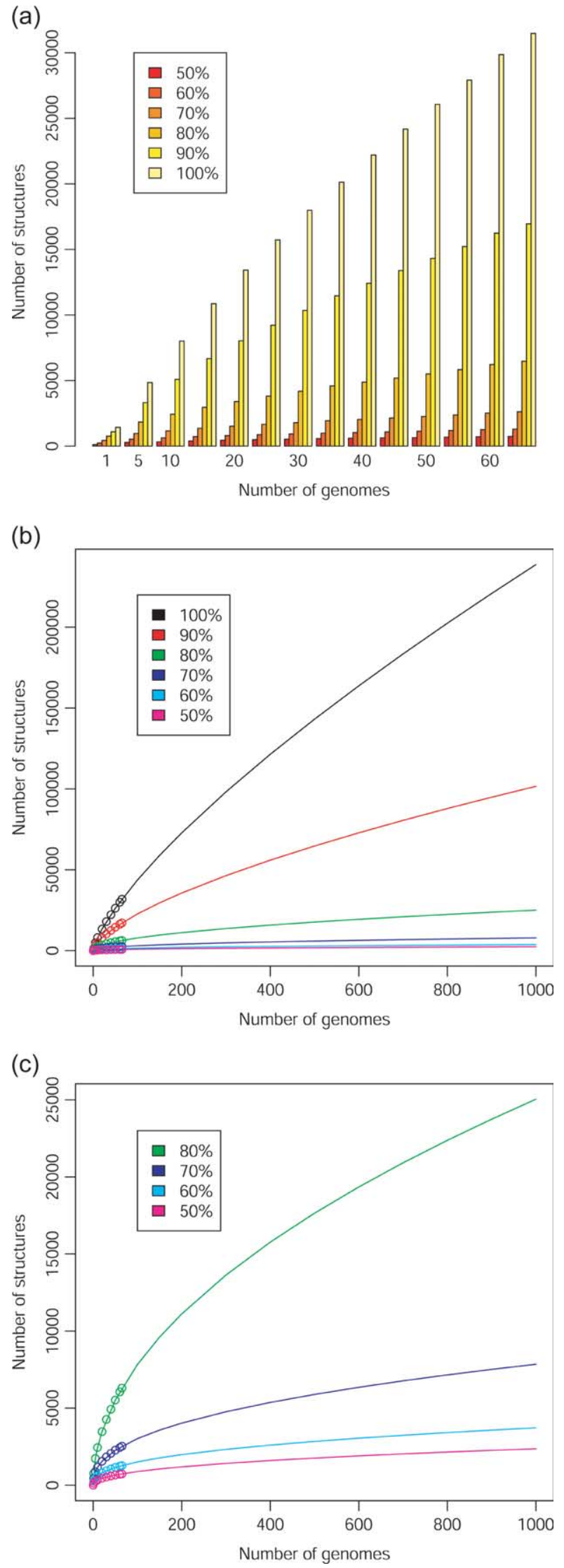

Figure 8. (a) Number of families with representative structures needed to provide structural coverage for different fractions of protein domains, as a function of the number of fully sequenced genomes. The lower the families already have representative structures.) A total of 1000 structures will complete coverage for all non-membrane families with more than ten members, covering $80 \%$ of the domains in these genomes.

\section{Achievable structural coverage for 1000 genomes}

We now examine how many structures will be needed to achieve a given level of protein coverage, as the number of fully sequenced genomes grows. For that purpose, an extrapolation procedure similar to that described earlier was used. A genome was picked at random from the set of 67 . The number of families was then calculated for that genome alone. The number of structures needed to obtain coverage of various fractions of all the proteins in that genome was calculated, assuming structures for the largest families are obtained first. Another genome was then selected randomly, and the number of structures needed to obtain various fractions of domain coverage for the two genomes was calculated, and so on, up to 65 genomes. The simulation was repeated 100 times, and the results averaged, to remove bias in genome order.

Figure 8(a) shows the results. Here, 100\% coverage implies models for all domains in all families, $90 \%$ coverage implies that $90 \%$ of the domains will have models, and so forth. The general trend is that the lower the domain coverage required, the slower the growth of the number of structures needed, as a function of the number of genomes. The growth rates for $80 \%$ and $90 \%$ coverage are already decreasing when 65 genomes are considered, and growth has almost ceased for $50 \%$ and $60 \%$ coverage. Figure 8(b) shows the estimates for up to 1000 genomes, based on log-linear models. At that stage, less than half of the number of structures are needed for $90 \%$ coverage as for $100 \%$, and the growth rate for $70 \%$ or lower coverage is slow. Figure 8(c) shows an expansion of the region below $80 \%$ coverage. Representative structures for about 8000 families will provide $70 \%$ coverage of all the domains in 1000 genomes. This is a reasonable expectation for the next decade, given the rate of accumulation of new experimental structures.

domain coverage required, the slower the growth in the number of families. (b) Projection of the number of families with representative structures needed to obtain structural coverage of different fractions of protein domains, up to 1000 genomes. A total of 250,000 structures would be required to obtain $100 \%$ coverage of these families, but $90 \%$ coverage would be obtained for less than half of that number. (c) Expansion of (b) for coverage between $50 \%$ and $80 \%$. For 1000 genomes, approximately 8000 structures are needed to provide $70 \%$ domain coverage, achievable in the next decade, considering the rate of accumulation of solved structures. 


\section{Discussion}

A principal goal of structure genomics is to obtain structures for a large fraction of naturally occurring proteins. This goal can be achieved by experimentally determining at least one structure for each protein family and building structure models for all other proteins, using comparative modeling methods. ${ }^{55}$ The minimum number of experimental structures required for complete structural coverage of protein space is then equal to the number of apparent protein families. In a previous study, ${ }^{8}$ we estimated this number by analyzing PfamA families, ${ }^{24}$ and making a very simple extrapolation of likely future growth in the number of families.

In the present study, we have based the analysis on all families in a set of fully sequenced prokaryotic genomes, rather than the contents of PfamA. A major difference is the inclusion of all proteins, not just those in the larger families typically collected in PfamA. With this more realistic view of the protein universe, we find there are a very large number of such small families: for the set of 67 genomes analyzed, there are 25,802 families with only one or two members, out of a total of 31,874 . Overall, there is an approximately power law relationship between the number of families and family size. In a comprehensive analysis of five eukaryotic genomes, Liu et al. also found a large number of small families, unique to that kingdom. ${ }^{37}$

Use of complete genome sequence sets has also allowed us to use a more realistic extrapolation method, in order to estimate the future growth in the number of families, as the number of fully sequenced genomes grows. We find that when 1000 genomes are available, there will be about 250,000 detectable protein families. Further, the number of families will still be growing at that point.

The large number of families makes it clear that complete structural coverage of protein space will not be possible in the near future. Nevertheless, it will be possible to obtain structural models for a large fraction of proteins. This is because most proteins belong to large families; for the 67 sequenced genomes, $88 \%$ of the proteins fall into just 6072 families. Further, the extrapolation model shows that this trend will continue, so that, considering all sequences, $80 \%$ structural coverage of the proteins in 1000 genomes can be obtained with 25,000 structures, and 70\% coverage with 8000 . The primary conclusion from this work is that a strategy of obtaining structural representatives for the largest families first will lead to a large fraction of structural coverage of protein space within the next decade. This strategy will also lead to early structural coverage of the families that perform more universal biological functions, and will provide the most leverage of experimental effort, by creating models for the largest number of proteins from each experimental structure. We envisage that when structures for proteins in small families are needed, they will typically be obtained one at a time, using conventional structural biology, rather than high-throughput methods.

The number of apparent protein families depends on the effectiveness of each of the steps in building them. There are three keys steps in our procedure. The first step uses PSI-BLAST to search for relatives of each protein. Other methods, in particular welltuned hidden Markov models ${ }^{51}$ and profile-profile methods, ${ }^{27-29}$ are more sensitive for this purpose. ${ }^{54,27}$ At low false positive rates, the later merging step compensates for the relative insensitivity of PSI-BLAST.

The second step of family building is parsing of proteins into domains. We have used a sequence profile-based approach, relying on the fact that the most insertions and deletions occur between domains. ${ }^{16,21}$ We apply the procedure very conservatively to minimize splitting within domains. As a consequence, this step has many false negatives; it does not split at many domain boundaries that are obvious at the structure level. Additional procedures might further improve our method: mapping known structural domains and PfamA domains onto the proteins. We have not done that, because the majority of these families have not been studied structurally, and many are not in PfamA, so that use of these signals may distort the choice of parameters for family building.

The third step in family building is merging lists of related domains and filtering out redundant entries, to create domain families. As noted earlier, over-merging is a well known problem in protein family building; a small number of incorrect entries in the initial lists of relatives can easily lead to substantial over-merging. To avoid this, we use a procedure that requires an increasing number of common entries as a function of alignment size.

The rules for merging and other steps were tuned by reconstructing a set of PfamA domains from the corresponding full-length sequences, and comparing the generated families with the PfamA ones. The final procedure was benchmarked by comparing pairwise relationships within a set of generated families with those in a set of SCOP superfamilies. While these testing methods are very useful, they are not ideal. PfamA is a sequencebased family set, and so omits a large number of evolutionary relationships (placing related proteins in different families). A more sensitive method may therefore appear to have an excessively large number of false positives, and consequently may be detuned to reduce these. PfamA also focuses on larger families, whereas the genome data are dominated by smaller families. As a result, a better method for PfamA may not necessarily be optimum on genome data, and performance may be different from that suggested by quality measures on PfamA. Similarly, SCOP contains only proteins with known structure, and these may be unrepresentative of proteins in genomes as a whole; for example, not including proteins with significant inherent disorder, and under-representing proteins that form 
part of complexes. Nevertheless, PfamA and SCOP are probably the best training and test sets available. As in most of computational biology, the lack of a gold standard for methods development and evaluation is an inherent limitation.

According to our and other benchmarking, at a $1 \%$ ratio of false positives versus true positives, only about $30 \%$ of the pairwise evolutionary relationships implied by structure can be recovered with present sequence comparison methods. A consequence is that families built with those methods do not approximate independent evolutionary lines. As more structures are available, the number of families will decrease very substantially, because of merging on the basis of structural similarity, rather than sequence. For the purposes of structural genomics, a single representative structure for very large families containing very remote relatives is not particularly desirable. As the remoteness of the relationship between proteins increases, the quality of a model built on the basis of a relative with an experimental structure decreases. In particular, a substantial fraction of residues (up to $50 \%$ ) will have no equivalent in the modeling template. ${ }^{56}$ Thus, although families generated from sequence relationships are suboptimal from an evolutionary standpoint, they are very suitable for structural genomics.

Contrary to our earlier expectation, ${ }^{8}$ the number of apparent singletons and other small families will continue to increase. Siew \& Fischer also found the number of singletons is steadily growing, though the percentage of singletons as a fraction of all sequences is decreasing. ${ }^{38}$ Because of the limited sensitivity of sequence methods, it is not possible to judge the biological significance of this at present. Many singletons may, in fact, have unrelated folds, as one estimate of the total number of folds suggests. ${ }^{57}$ Or, most may turn out to be members of larger superfamilies, too remotely related for sequence methods to detect. A larger set of experimental structures of small families will settle this issue.

This work assessed how many experimental structures will be needed to provide models of a given fraction of all naturally occurring domains, based on one representative structure per family. Under this strategy, the majority of structures will be domain models, based on a single experimental structure within a protein family. While such a structure set will revolutionize our view of proteins in many ways, it is only the first step in providing complete structural information for natural proteins. Many proteins are multi-domain, particularly in higher eukaryotes, ${ }^{58,59}$ and the function of a domain assembly is not always a simple combination of that in the constituent domains. ${ }^{59}$ Generating reliable multi-domain structures will sometimes involve docking of domain models, requiring improvements in computational methods, or further experimental structures. Second, the relationships within families on which models will be based are often fairly distant, with levels of sequence identity well below 30\%. Models based on such sequence relationships contain substantial errors, arising primarily from mistakes in aligning the sequence of interest with those of available templates, and because significant parts of the structures will differ from that of the templates. $^{11}$ Nevertheless, these low accuracy models will be adequate for establishing membership of a superfamily, and thus useful for a variety of purposes, including providing approximate molecular function information, guiding sitedirected mutagenesis experiments, and choosing likely antigenic peptides. Other uses, such as identification of ligand specificity ${ }^{60}$ and interpretation of the effect of disease-related mutations, ${ }^{61}$ require greater accuracy, possible only by modeling against a template with $30 \%$ or greater sequence identity. Comprehensive structural information at that level will require many more structures.

\section{Acknowledgements}

We thank Eugene Melamud for help with many aspects of data management. This work was supported, in part, by NIH grant GM57890.

\section{References}

1. Berman, H. M. \& Westbrook, J. D. (2004). The impact of structural genomics on the protein data bank. Am. J. Pharmacogenomics, 4, 247-252.

2. Browne, W. J., North, A. C., Phillips, D. C., Brew, K., Vanaman, T. C. \& Hill, R. L. (1969). A possible threedimensional structure of bovine alpha-lactalbumin based on that of hen's egg-white lysozyme. J. Mol. Biol. 42, 65-86.

3. Brenner, S. E. (2001). A tour of structural genomics. Naure Rev. Genet. 2, 801-809.

4. Wolf, Y. I., Grishin, N. V. \& Koonin, E. V. (2000). Estimating the number of protein folds and families from complete genome data. J. Mol. Biol. 299, 897-905.

5. Orengo, C. A., Todd, A. E. \& Thornton, J. M. (1999). From protein structure to function. Curr. Opin. Struct. Biol. 9, 374-382.

6. Sternberg, M. J., Bates, P. A., Kelley, L. A. \& MacCallum, R. M. (1999). Progress in protein structure prediction: assessment of CASP3. Curr. Opin. Struct. Biol. 9, 368-373.

7. Liu, J. \& Rost, B. (2002). Target space for structural genomics revisited. Bioinformatics, 18, 922-933.

8. Vitkup, D., Melamud, E., Moult, J. \& Sander, C. (2001). Completeness in structural genomics. Nature Struct. Biol. 8, 559-566.

9. Tramontano, A. \& Morea, V. (2003). Assessment of homology-based predictions in CASP5. Proteins: Struct. Funct. Genet. 53, 352-368.

10. Baker, D. \& Sali, A. (2001). Protein structure prediction and structural genomics. Science, 294, 93-96.

11. Venclovas, C., Zemla, A., Fidelis, K. \& Moult, J. (2003). Assessment of progress over the CASP experiments. Proteins: Struct. Funct. Genet. 53, 585-595. 
12. Nagano, N., Orengo, C. A. \& Thornton, J. M. (2002). One fold with many functions: the evolutionary relationships between TIM barrel families based on their sequences, structures and functions. J. Mol. Biol. 321, 741-765.

13. Dayhoff, M. O. (1976). The origin and evolution of protein superfamilies. Fed. Proc. Fed. Am. Soc. Expt. Biol. 35, 2132-2138.

14. Haft, D. H., Selengut, J. D. \& White, O. (2003). The TIGRFAMs database of protein families. Nucl. Acids Res. 31, 371-373.

15. Servant, F., Bru, C., Carrere, S., Courcelle, E., Gouzy, J., Peyruc, D. \& Kahn, D. (2002). ProDom: automated clustering of homologous domains. Brief Bioinform. 3, 246-251.

16. Gracy, J. \& Argos, P. (1998). Automated protein sequence database classification. II. Delineation of domain boundaries from sequence similarities. Bioinformatics, 14, 174-187.

17. Gracy, J. \& Argos, P. (1998). Automated protein sequence database classification. I. Integration of compositional similarity search, local similarity search, and multiple sequence alignment. Bioinformatics, 14, 164-173.

18. Enright, A. J., Van Dongen, S. \& Ouzounis, C. A. (2002). An efficient algorithm for large-scale detection of protein families. Nucl. Acids Res. 30, 1575-1584.

19. Mohseni-Zadeh, S., Brezellec, P. \& Risler, J. L. (2004). Cluster-C, an algorithm for the large-scale clustering of protein sequences based on the extraction of maximal cliques. Comput. Biol. Chem. 28, 211-218.

20. Gough, J. (2002). The SUPERFAMILY database in structural genomics. Acta Crystallog. sect. D, 58, 18971900.

21. Heger, A. \& Holm, L. (2001). Picasso: generating a covering set of protein family profiles. Bioinformatics, 17, 272-279.

22. Yona, G., Linial, N. \& Linial, M. (2000). ProtoMap: automatic classification of protein sequences and hierarchy of protein families. Nucl. Acids Res. 28, $49-55$.

23. Wu, C. H., Xiao, C., Hou, Z., Huang, H. \& Barker, W. C. (2001). iProClass: an integrated, comprehensive and annotated protein classification database. Nucl. Acids Res. 29, 52-54.

24. Sonnhammer, E. L., Eddy, S. R., Birney, E., Bateman, A. \& Durbin, R. (1998). Pfam: multiple sequence alignments and HMM-profiles of protein domains. Nucl. Acids Res. 26, 320-322.

25. Hubbard, T. J., Murzin, A. G., Brenner, S. E. \& Chothia, C. (1997). SCOP: a structural classification of proteins database. Nucl. Acids Res. 25, 236-239.

26. Andreeva, A., Howorth, D., Brenner, S. E., Hubbard, T. J., Chothia, C. \& Murzin, A. G. (2004). SCOP database in 2004: refinements integrate structure and sequence family data. Nucl. Acids Res. 32, D226-D229.

27. Ohlson, T., Wallner, B. \& Elofsson, A. (2004). Profileprofile methods provide improved fold-recognition: a study of different profile-profile alignment methods. Proteins: Struct. Funct. Genet. 57, 188-197.

28. Edgar, R. C. \& Sjolander, K. (2004). COACH: profileprofile alignment of protein families using hidden Markov models. Bioinformatics, 20, 1309-1318.

29. Sadreyev, R. \& Grishin, N. (2003). COMPASS: a tool for comparison of multiple protein alignments with assessment of statistical significance. J. Mol. Biol. 326, 317-336.

30. Brenner, S. E., Chothia, C. \& Hubbard, T. J. (1997).
Population statistics of protein structures: lessons from structural classifications. Curr. Opin. Struct. Biol. 7, 369-376.

31. Chothia, C. (1992). Proteins. One thousand families for the molecular biologist. Nature, 357, 543-544.

32. Holm, L. \& Sander, C. (1996). Mapping the protein universe. Science, 273, 595-603.

33. Wang, Z. X. (1998). A re-estimation for the total numbers of protein folds and superfamilies. Protein Eng. 11, 621-626.

34. Zhang, C. \& DeLisi, C. (1998). Estimating the number of protein folds. J. Mol. Biol. 284, 1301-1305.

35. Govindarajan, S., Recabarren, R. \& Goldstein, R. A. (1999). Estimating the total number of protein folds. Proteins: Struct. Funct. Genet. 35, 408-414.

36. Grant, A., Lee, D. \& Orengo, C. (2004). Progress towards mapping the universe of protein folds. Genome Biol. 5, 107.

37. Liu, J., Hegyi, H., Acton, T. B., Montelione, G. T. \& Rost, B. (2004). Automatic target selection for structural genomics on eukaryotes. Proteins: Struct. Funct. Genet. 56, 188-200.

38. Siew, N. \& Fischer, D. (2003). Analysis of singleton ORFans in fully sequenced microbial genomes. Proteins: Struct. Funct. Genet. 53, 241-251.

39. Altschul, S. F., Madden, T. L., Schaffer, A. A., Zhang, J., Zhang, Z., Miller, W. \& Lipman, D. J. (1997). Gapped BLAST and PSI-BLAST: a new generation of protein database search programs. Nucl. Acids Res. 25, 3389-3402.

40. Altschul, S. F. \& Koonin, E. V. (1998). Iterated profile searches with PSI-BLAST-a tool for discovery in protein databases. Trends Biochem. Sci. 23, 444-447.

41. Wootton, J. C. (1994). Non-globular domains in protein sequences: automated segmentation using complexity measures. Comput. Chem. 18, 269-285.

42. Liu, J. \& Rost, B. (2004). CHOP: parsing proteins into structural domains. Nucl. Acids Res. 32, W569-W571.

43. Liu, J. \& Rost, B. (2004). Sequence-based prediction of protein domains. Nucl. Acids Res. 32, 3522-3530.

44. Pipenbacher, P., Schliep, A., Schneckener, S., Schonhuth, A., Schomburg, D. \& Schrader, R. (2002). ProClust: improved clustering of protein sequences with an extended graph-based approach. Bioinformatics, 18, S182-S191.

45. Yona, G., Linial, N. \& Linial, M. (1999). ProtoMap: automatic classification of protein sequences, a hierarchy of protein families, and local maps of the protein space. Proteins: Struct. Funct. Genet. 37, 360-378.

46. Kahsay, R. Y., Wang, G., Dongre, N., Gao, G. \& Dunbrack, R. L., Jr (2002). CASA: a server for the critical assessment of protein sequence alignment accuracy. Bioinformatics, 18, 496-497.

47. Brenner, S. E., Chothia, C. \& Hubbard, T. J. (1998). Assessing sequence comparison methods with reliable structurally identified distant evolutionary relationships. Proc. Natl Acad. Sci. USA, 95, 6073-6078.

48. Park, J., Teichmann, S. A., Hubbard, T. \& Chothia, C. (1997). Intermediate sequences increase the detection of homology between sequences. J. Mol. Biol. 273, 349-354.

49. Park, J., Holm, L., Heger, A. \& Chothia, C. (2000). RSDB: representative protein sequence databases have high information content. Bioinformatics, 16, 458-464.

50. Enright, A. J. \& Ouzounis, C. A. (2000). GeneRAGE: a robust algorithm for sequence clustering and domain detection. Bioinformatics, 16, 451-457. 
51. Karplus, K. \& Hu, B. (2001). Evaluation of protein multiple alignments by SAM-T99 using the BAliBASE multiple alignment test set. Bioinformatics, 17, 713-720.

52. Krogh, A., Larsson, B., von Heijne, G. \& Sonnhammer, E. L. (2001). Predicting transmembrane protein topology with a hidden Markov model: application to complete genomes. J. Mol. Biol. 305, 567-580.

53. Marchler-Bauer, A., Panchenko, A. R., Shoemaker, B. A., Thiessen, P. A., Geer, L. Y. \& Bryant, S. H. (2002). CDD: a database of conserved domain alignments with links to domain three-dimensional structure. Nucl. Acids Res. 30, 281-283.

54. Park, J., Karplus, K., Barrett, C., Hughey, R., Haussler, D., Hubbard, T. \& Chothia, C. (1998). Sequence comparisons using multiple sequences detect three times as many remote homologues as pairwise methods. J. Mol. Biol. 284, 1201-1210.

55. Marti-Renom, M. A., Stuart, A. C., Fiser, A., Sanchez,
R., Melo, F. \& Sali, A. (2000). Comparative protein structure modeling of genes and genomes. Annu. Rev. Biophys. Biomol. Struct. 29, 291-325.

56. Chothia, C. \& Lesk, A. M. (1986). The relation between the divergence of sequence and structure in proteins. EMBO J. 5, 823-826.

57. Coulson, A. F. \& Moult, J. (2002). A unifold, mesofold, and superfold model of protein fold use. Proteins: Struct. Funct. Genet. 46, 61-71.

58. Vogel, C., Bashton, M., Kerrison, N. D., Chothia, C. \& Teichmann, S. A. (2004). Structure, function and evolution of multidomain proteins. Curr. Opin. Struct. Biol. 14, 208-216.

59. Bashton, M. \& Chothia, C. (2002). The geometry of domain combination in proteins. J. Mol. Biol. 315, 927-939.

60. DeWeese-Scott, C. \& Moult, J. (2004). Molecular modeling of protein function regions. Proteins: Struct. Funct. Genet. 55, 942-961.

61. Wang, Z. \& Moult, J. (2001). SNPs, protein structure, and disease. Hum. Mutat. 17, 263-270.

Edited by B. Honig

(Received 25 March 2005; received in revised form 18 August 2005; accepted 24 August 2005) Available online 9 September 2005 